\title{
A characterization of curves according to parallel transport frame in Euclidean n-space $\mathbb{E}^{n}$
}

\author{
Sezgin Buyukkutuk, Ilim Kisi and Gunay Ozturk \\ Department of Mathematics,Kocaeli University, Kocaeli, Turkey
}

Received: 17 May 2016, Accepted: 17 June 2016

Published online: 27 April 2017.

\begin{abstract}
The position vector of a regular curve in Euclidean $n$-space $\mathbb{E}^{n}$ can be written as a linear combination of its parallel transport vectors. In the present study, we characterize such curves in terms of their curvature functions. Further, we obtain some results of constant ratio, $T$-constant and $N$-constant type curves in $\mathbb{E}^{n}$.
\end{abstract}

Keywords: Parallel transport frame, position vector, constant-ratio curves.

\section{Introduction}

Rectifying curves in Euclidean 3-space $\mathbb{E}^{3}$ were introduced by B. Y. Chen in [5] as space curves whose position vectors (denoted also by $x$ ) lie in their rectifying planes, spanned by the tangent and the binormal normal vector fields $T(s)$ and $N_{2}(s)$ of the curve. In the same paper, B. Y. Chen gave a simple characterization of rectifying curves.

In [11], Ilarslan and Nesovic considered the rectifying curve in Euclidean 4-space $\mathbb{E}^{4}$. They characterized the rectifying curves given by the equation

$$
x(s)=\lambda(s) T(s)+\mu(s) N_{2}(s)+v(s) N_{3}(s),
$$

for some differentiable functions $\lambda(s), \mu(s)$ and $v(s)$. Also in [8], the authors characterized the rectifying curves in n-dimensional Euclidean space.

For a regular curve $x(s)$, the position vector $x$ can be decomposed into its tangential and normal components at each point, i.e., $x=x^{T}+x^{N}$. A curve $x(s)$ with $\kappa_{1}(s)>0$ is said to be of constant ratio if the ratio $\left\|x^{T}\right\|:\left\|x^{N}\right\|$ is constant on $x(I)$ where $\left\|x^{T}\right\|$ and $\left\|x^{N}\right\|$ denote the length of $x^{T}$ and $x^{N}$, respectively [4]. Clearly a curve $x$ in $\mathbb{E}^{n}$ is of constant ratio if and only if $x^{T}=0$ or $\left\|x^{T}\right\|:\|x\|$ is constant. The distance function $\rho=\|x\|$ satisfies $\|\operatorname{grad} \rho\|=c$ for some constant $c$ if and only if we have $\left\|x^{T}\right\|=c\|x\|$. In particular, if $\|\operatorname{grad} \rho\|=c$, then $c \in[0,1]$. In the same paper, B. Y. Chen gave a classification of constant ratio curves in Euclidean space.

A curve in $\mathbb{E}^{n}$ is called $T$-constant (resp. $N$-constant) if the tangential component $x^{T}$ (resp. the normal component $x^{N}$ ) of its position vector $x$ is of constant length [6]. Recently the present authors study with the constant ratio curves in Euclidean 4-space $\mathbb{E}^{4}$ in [3]. For more details see also [2,?,?].

The Frenet frame is constructed for the curve of 3-time continuously differentiable non-degenerate curves. But, curvature may vanish at some points on the curve. That is, second derivative of the curve may be zero. In this situation, 
we need an alternative frame in $\mathbb{E}^{3}$. Therefore in [1], Bishop defined a new frame for a curve and he called it Bishop frame which is well defined even if the curve's second derivative vanishes in 3-dimensional Euclidean space. In Euclidean $n$-space $\mathbb{E}^{n}$, we have the same problem, that is, one of the $i-$ th $(1<i<n)$ derivative of the curve may vanish. In [13], the authors gave parallel transport frame of a curve in $n$-dimensional Euclidean space.

In the present study, we consider a curve in Euclidean $n$-space $\mathbb{E}^{n}$ as a curve whose position vector can be written as a linear combination of its parallel transport frame. Then its position vector satisfies the parametric equation

$$
x(s)=m_{0}(s) T(s)+m_{1}(s) M_{1}(s)+\ldots+m_{i}(s) M_{i}(s)+\ldots+m_{n-1}(s) M_{n-1}(s),
$$

for some differentiable functions, $m_{i}(s), 0 \leq i \leq n-1$, where $\left\{T, M_{1}, \ldots, M_{n-1}\right\}$ is its parallel transport frame. We characterize such curves in terms of their curvature functions $k_{i}(s), 0 \leq i \leq n-1$ and give the necessary and sufficient conditions for such curves to become contant ratio, $T$-constant and $N$-constant curves in $\mathbb{E}^{n}$.

\section{Basic notations and known results}

Analogous as for a space curve, for an arclength parameterized curve $x: I \subset \mathbb{R} \rightarrow \mathbb{E}^{n}$ that is $n$ times continuously differentiable, one can construct a Frenet frame, $T, N_{1}, . . N_{i}, . . N_{n-1}$ that satisfies the equations (see, [9]):

$$
\begin{aligned}
T^{\prime}(s) & =\kappa_{1}(s) N_{1}(s), \\
N_{1}^{\prime}(s) & =-\kappa_{1}(s) T(s)+\kappa_{2}(s) N_{2}(s), \\
N_{2}^{\prime}(s) & =-\kappa_{2}(s) N_{1}(s)+\kappa_{3}(s) N_{3}(s), \\
N_{i}^{\prime}(s) & =-\kappa_{i}(s) N_{i-1}(s)+\kappa_{i+1}(s) N_{i+1}(s), \\
N_{n-1}^{\prime}(s) & =-\kappa_{n-1}(s) N_{n-2}(s) .
\end{aligned}
$$

If the curve $x$ is not arclength parameterized, then the right-hand sides of the equations (3) must be multiplied by the speed $v$ of $x$.

The functions $\kappa_{i}$ for $i \in\{1,2, \ldots, n-1\}$ are the curvatures of the curve. All $\kappa_{i}$ are positive for $i \in\{1,2, \ldots, n-2\}$.

Further, let $x$ be a unit speed curve in Euclidean $n$-space $\mathbb{E}^{n}$ with the tangent vector $T(s)$. One can choose any convenient arbitrary basis which consists of relatively parallel vector fields $M_{1}(s), M_{2}(s), \ldots, M_{n-1}(s)$ which are perpendicular to $T(s)$ at each point. The parallel transport frame equations are (see [13])

$$
\left[\begin{array}{c}
T^{\prime} \\
M_{1}^{\prime} \\
M_{2}^{\prime} \\
\vdots \\
M_{n-1}^{\prime}
\end{array}\right]=\left[\begin{array}{ccccc}
0 & -k_{1} & -k_{2} & \ldots & -k_{n-1} \\
k_{1} & 0 & \ldots & \ldots & \ldots \\
k_{2} & 0 & \ldots & \ldots & \ldots \\
\vdots & \vdots & \vdots & \vdots & \vdots \\
k_{n-1} & 0 & \ldots & \ldots & \ldots
\end{array}\right]\left[\begin{array}{c}
T \\
M_{1} \\
M_{2} \\
\vdots \\
M_{n-1}
\end{array}\right]
$$

where $k_{i}$ are principle curvature functions according to parallel transport frame of the curve $x$.

\section{Characterization of curves according to parallel transport frame in $\mathbb{E}^{n}$}

In the present section, we consider unit speed curves with Bishop curvatures $k_{i}(s)$ for $i \in\{1,2, \ldots, n-1\}$. By definition, the position vector of the curve (also defined by $x$ ) satisfies the vectorial equation (2) for some differentiable functions 
$m_{i}(s), 0 \leq i \leq n-1$. By taking the derivative of (2) with respect to arclength parameter $s$ and using the parallel transport frame equations (4), we obtain

$$
x^{\prime}(s)=\left(m_{0}^{\prime}(s)+k_{1}(s) m_{1}(s)+\ldots+k_{i}(s) m_{i}(s)+\ldots+k_{n-1}(s) m_{n-1}(s)\right) T(s)+\sum_{i=1}^{n-1}\left(m_{i}^{\prime}(s)-k_{i}(s) m_{0}(s)\right) M_{i}(s) .
$$

It follows that

$$
\begin{aligned}
m_{0}^{\prime}+k_{1} m_{1}+\ldots+k_{i} m_{i}+\ldots+k_{n-1} m_{n-1} & =1 \\
m_{1}^{\prime}-k_{1} m_{0} & =0 \\
m_{i}^{\prime}-k_{i} m_{0} & =0,(2 \leq i \leq n-1) .
\end{aligned}
$$

Theorem 1. Let $x: I \subset \mathbb{R} \rightarrow \mathbb{E}^{n}$ be a unit speed curve in $\mathbb{E}^{n}$ with the vectorial equation (2). If $x$ has constant curvatures $\left(k_{i}=\right.$ constant), then the position vector $x$ is given by the curvature functions

$$
\begin{aligned}
& m_{0}(s)=c_{1} \cos \lambda s+c_{2} \sin \lambda s \\
& m_{1}(s)=k_{1}\left(\frac{c_{1} \sin \lambda s-c_{2} \cos \lambda s}{\lambda}\right)+c_{3}, \\
& m_{i}(s)=k_{i}\left(\frac{c_{1} \sin \lambda s-c_{2} \cos \lambda s}{\lambda}\right)+c_{i+2},(2 \leq i \leq n-1),
\end{aligned}
$$

where $c_{i},(1 \leq i \leq n+1)$ are integral constants and $\lambda=\sqrt{k_{1}^{2}+\ldots+k_{n-1}^{2}}$ is a real constant.

Proof. Let $x$ has constant curvatures ( $k_{i}=$ constant), then by the use of the equations (6), we get

$$
m_{0}^{\prime \prime}=-\left(k_{1}^{2}+\ldots+k_{n-1}^{2}\right) m_{0}
$$

One can show that the equation (8) has a non-trivial solution

$$
m_{0}=c_{1} \cos \sqrt{k_{1}^{2}+\ldots+k_{n-1}^{2}} s+c_{2} \sin \sqrt{k_{1}^{2}+\ldots+k_{n-1}^{2}} s .
$$

Further, substituting this solution into (6) and integrating these equations, we get the result.

\section{1 - constant curves}

Definition 1. Let $x: I \subset \mathbb{R} \rightarrow \mathbb{E}^{n}$ be a unit speed curve in $\mathbb{E}^{n}$. If $\left\|x^{T}\right\|$ is constant, then $x$ is called a T-constant curve. For a $T$-constant curve $x$, either $\left\|x^{T}\right\|=0$ or $\left\|x^{T}\right\|=\lambda$ for some non-zero smooth function $\lambda$ (see,[6]). Further, a T-constant curve $x$ is called first kind if $\left\|x^{T}\right\|=0$, otherwise second kind [10].

Theorem 2. Let $x: I \subset \mathbb{R} \rightarrow \mathbb{E}^{n}$ be a curve with nonzero curvatures $k_{i}(i=1, \ldots, n-1)$ according to parallel transport frame in Euclidean $n-$ space $\mathbb{E}^{n}$. Then $x$ lies on a sphere if and only if

$$
\sum_{i=1}^{n-1} c_{i} k_{i}(s)=1
$$

where $c_{i}(i=1, \ldots, n-1)$ are non-zero constants.

Proof. Let $x$ be a curve on a sphere with the center $P$ and radius $r$, then $\langle x-P, x-P\rangle=r^{2}$. Differentiating this equation, we obtain that $\langle T, x-P\rangle=0$. We can write $x-P=c_{1} M_{1}+. .+c_{i} M_{i}+. .+c_{n-1} M_{n-1}$ for some functions $c_{i}(i=1, . ., n-1)$ 
and where $c_{1}^{\prime}=\left\langle x-P, M_{1}\right\rangle^{\prime}=\left\langle T, M_{1}\right\rangle+\left\langle k_{1} T, x-P\right\rangle=0$. Hence, $c_{1}$ is a constant function. Similarly, we can easily say that all of the functions $c_{i}(i=1, . ., n-1)$ are constants. Then differentiating the equation $\langle T, x-P\rangle$, we get

$$
\left\langle-\left(k_{1} M_{1}+\ldots+k_{n-1} M_{n-1}\right), x-P\right\rangle+\langle T, T\rangle=0 .
$$

Consequently, the curvatures $k_{i}(i=1, . ., n-1)$ of the curve have the linear relation

$$
-\sum_{i=1}^{n-1} c_{i} k_{i}+1=0
$$

Conversely, we suppose that

$$
-\left(c_{1} k_{1}+\ldots+c_{n-1} k_{n-1}\right)+1=0 .
$$

If the center $P$ denoted by $P=x-c_{1} M_{1}-. .-c_{i} M_{i}-. .-c_{n-1} M_{n-1}$, then differentiating the last equation, we have $P^{\prime}=$ $T-\left(c_{1} k_{1}+\ldots+c_{n-1} k_{n-1}\right) T=0$. Thus, the center $P$ of the sphere is constant. Similarly, we show that $r^{2}=\langle x-P, x-P\rangle$ is constant. As a result of these, the curve $x$ lies on a sphere with center $P$ and radius $r$.

Corollary 1. Let $x: I \subset \mathbb{R} \rightarrow \mathbb{E}^{n}$ be a unit speed curve given with the parametrization (2) in $\mathbb{E}^{n}$. Then $x$ is a T-constant curve of first kind if and only if $x$ lies on a sphere.

Proof.Let $x$ be a $T$-constant curve of first kind, then from the $i$-th equalities $(i=1 . . n-1)$ in (6), we get $m_{i}^{\prime}=0(i=$ $1, \ldots, n-1)$. Further substituting $m_{i}=c_{i}$ into the first equation, we get $\sum_{i=1}^{n-1} c_{i} k_{i}=1$. From Theorem 2 , we get the result.

Theorem 3. Let $x: I \subset \mathbb{R} \rightarrow \mathbb{E}^{n}$ be a unit speed curve in $\mathbb{E}^{n}$. $x$ is a $T$-constant curve of second kind if and only if

$$
\sum_{i=1}^{n-1} k_{i}(s) \int k_{i}(s) d s=\frac{1}{m_{0}}
$$

holds.

Proof. Let $x$ be a $T$-constant curve of second kind, then from (6), we get

$$
\sum_{i=1}^{n-1} k_{i} m_{i}=1
$$

Further, integrating the $i-$ th equalities $(i=2 \ldots n-1)$ in (6) and substituting these values into (9), we get the result.

Corollary 2. Let $x: I \subset \mathbb{R} \rightarrow \mathbb{E}^{n}$ be a unit speed curve in $\mathbb{E}^{n}$. If $x$ is a $T$-constant curve of second kind, the curvature functions $m_{i}$ of the curve $x$ satisfy the equation

$$
2 m_{0} s+c=\sum_{i=1}^{n-1} m_{i}^{2}
$$

where $c$ is an integral constant.

Proof. Let $x$ be a $T$-constant curve of second kind, from the $i$-th equalities $(i=2 \ldots n-1)$ in (6), we get

$$
k_{i}=\frac{m_{i}^{\prime}}{m_{0}},(i=1 \ldots n-1) .
$$


Substituting these values into the first equation in (6), we obtain the differential equation

$$
\sum_{i=1}^{n-1} m_{i} m_{i}^{\prime}=m_{0}
$$

which has the solution (10).

Theorem 4. Let $x: I \subset \mathbb{R} \rightarrow \mathbb{E}^{n}$ be a $T$-constant curve of second kind. Then the distance function $\rho=\|x\|$ satisfies

$$
\rho= \pm \sqrt{2 \lambda s+c}
$$

for some real constants $c$ and $\lambda=m_{0}$.

Proof. Differentiating the squared distance function $\rho^{2}=\langle x(s), x(s)\rangle$ and using (2), we get $\rho \rho^{\prime}=m_{0}$. If $x$ is a $T$-constant curve of second kind, then by definition the curvature function $m_{0}(s)$ of $x$ is constant. It is easy to show that this differential equation has a nontrivial solution (11).

\section{$3.2 N$-constant curves}

Definition 2. Let $x: I \subset \mathbb{R} \rightarrow \mathbb{E}^{n}$ be a unit speed curve in $\mathbb{E}^{n}$. If $\left\|x^{N}\right\|$ is constant, then $x$ is called a $N$-constant curve. For a $N$-constant curve $x$, either $\left\|x^{N}\right\|=0$ or $\left\|x^{N}\right\|=\mu$ for some non-zero smooth function $\mu$ (see, [6]). Further, a $N$-constant curve $x$ is called first kind if $\left\|x^{N}\right\|=0$. otherwise second kind [10].

Hence, for a $N$-constant curve $x$ in $\mathbb{E}^{n}$

$$
\left\|x^{N}(s)\right\|^{2}=m_{1}^{2}(s)+m_{2}^{2}(s)+\ldots+m_{n-1}^{2}(s)
$$

becomes a constant function. Therefore, by differentiation

$$
m_{1} m_{1}^{\prime}+m_{2} m_{2}^{\prime}+\ldots+m_{n-1} m_{n-1}^{\prime}=0 .
$$

For the $N$-constant curves of first kind, we give the following result.

Proposition 1. Let $x: I \subset \mathbb{R} \rightarrow \mathbb{E}^{n}$ be a unit speed curve in $\mathbb{E}^{n}$. $x$ is a $N$-constant curve of first kind if and only if $x(I)$ is an open portion of a straight line.

Proof. Suppose that $x$ is a $N$-constant curve of first kind in $\mathbb{E}^{n}$, then the equality (12) holds. Further, if $x$ is of first kind, then from (12) $m_{1}=m_{2}=\ldots=m_{n-1}=0$ which implies that $k_{1}=k_{2}=\ldots=k_{n-1}=0$. Then the first Frenet curvature of the curve $x$ is zero. Hence, $x$ is a part of a straight line.

Further, for the $N$-constant curves of second kind, we obtain the following results.

Theorem 5. Let $x: I \subset \mathbb{R} \rightarrow \mathbb{E}^{n}$ be a unit speed curve in $\mathbb{E}^{n}$ and $s$ be an arclength function. If $x$ is a $N$-constant curve of second kind, then $x$ is a $T$-constant curve of first kind with the parametrization

$$
x(s)=\lambda_{1} M_{1}(s)+\lambda_{2} M_{2}(s)+\ldots+\lambda_{n-1} M_{n-1}(s),
$$

where $\lambda_{i}(i=1, \ldots, n-1)$ are real constants or the curve has the parametrization

$$
x(s)=(s+c) T(s)+\left(\int(s+c) k_{1}(s) d s\right) M_{1}(s)+\ldots+\left(\int(s+c) k_{n-1}(s) d s\right) M_{n-1}(s),
$$


where $c$ is real constant.

Proof. Let $x$ be a $N$-constant curve of second kind in $\mathbb{E}^{n}$, then from (6) and (13), we get $m_{0}\left(k_{1} m_{1}+\ldots k_{i} m_{i} \ldots+k_{n-1} m_{n-1}\right)=$ 0 . Hence, there are two possible cases; $m_{0}=0$ or $k_{1} m_{1}+\ldots k_{i} m_{i} \ldots+k_{n-1} m_{n-1}=0$. The first case with the equation (6) implies that $m_{i}=\lambda_{i}=$ const. Thus, $x$ is a $T$-constant curve of first kind with the parametrization (14). For the second case by the use of (6), we get

$$
\begin{aligned}
& m_{0}=s+c, \\
& m_{1}=\int(s+c) k_{1}(s) d s, \\
& m_{i}=\int(s+c) k_{i}(s) d s,(2 \leq i \leq n-1),
\end{aligned}
$$

which completes the proof of the theorem.

Theorem 6. Let $x: I \subset \mathbb{R} \rightarrow \mathbb{E}^{n}$ be a $N$-constant curve of second kind. Then the distance function $\rho=\|x\|$ satisfies

$$
\rho=\mp \sqrt{s^{2}+2 b s+d}
$$

for some constant functions $b, d$.

Proof. Differentiating the squared distance function $\rho^{2}=\langle x(s), x(s)\rangle$ and using (2), we get $\rho \rho^{\prime}=m_{0}$. If $x$ is a $N$-constant curve of second kind, then from the previous Theorem $m_{0}(s)=s+b$. It is easy to show that this differential equation has a nontrivial solution (15).

\subsection{Constant-ratio curves}

Definition 3. Let $x: I \subset \mathbb{R} \rightarrow \mathbb{E}^{n}$ be a unit speed regular curve in $\mathbb{E}^{n}$. Then the position vector $x$ can be decomposed into its tangential and normal components at each point:

$$
x=x^{T}+x^{N} .
$$

If the ratio $\left\|x^{T}\right\|:\left\|x^{N}\right\|$ is constant on $x(I)$, then $x$ is said to be of constant ratio, or equivalently $\left\|x^{T}\right\|:\|x\|=c=$ constant [4].

For a unit speed regular curve $x$ in $\mathbb{E}^{n}$, the gradient of the distance function $\rho=\|x(s)\|$ is given by

$$
\operatorname{grad} \rho=\frac{d \rho}{d s} T(s)=\frac{<x(s), T(s)>}{\|x(s)\|} T(s),
$$

where $T$ is the tangent vector field of $x$. The following results characterize constant-ratio curves.

Theorem 7. [7] Let $x: I \subset \mathbb{R} \rightarrow \mathbb{E}^{n}$ be a unit speed regular curve in $\mathbb{E}^{n}$. Then $x$ is of constant ratio with $\left\|x^{T}\right\|:\|x\|=c$ if and only if $\|\operatorname{grad} \rho\|=c$ which is constant. In particular, for a curve of constant ratio, we have $\|\operatorname{grad} \rho\|=c \leq 1$.

As a consequence of (16), the following result were obtained.

Theorem 8. [7] Let $x: I \subset \mathbb{R} \rightarrow \mathbb{E}^{n}$ be a unit speed regular curve in $\mathbb{E}^{n}$. Then $\|$ grad $\rho \|=c$ holds for a constant $c$ if and only if one of the following three cases occurs.

(i) $\|\operatorname{grad} \rho\|=0 \Longleftrightarrow x(I)$ is contained in a hypersphere centered at the origin. 
(ii) $\|\operatorname{grad} \rho\|=1 \Longleftrightarrow x(I)$ is an open portion of a line through the origin.

(iii) $\|\operatorname{grad} \rho\|=c \Longleftrightarrow \rho=\|x(s)\|=c s$, for $c \in(0,1)$.

The following result provides some simple characterization of constant ratio curves in $\mathbb{E}^{n}$. Observe that, this result is also valid in three and four dimensional cases (see, [2], [3]) .

Proposition 2. Let $x: I \subset \mathbb{R} \rightarrow \mathbb{E}^{n}$ be a unit speed curve in $\mathbb{E}^{n}$. Then $x$ is a constant-ratio curve if and only if

$$
\sum_{i=1}^{n-1}\left(k_{i}(s) \int s k_{i}(s) d s\right)=\frac{1-c^{2}}{c^{2}}
$$

holds.

Proof. Let $x$ be a curve of constant-ratio given with the arclength function $s$. Then, from the previous result, the distance function $\rho$ of $x$ satisfies the equality $\rho=\|x(s)\|=c s$ for some real constant $c$. Further, using (16), we get

$$
\|\operatorname{grad} \rho\|=\frac{<x(s), T(s)>}{\|x(s)\|}=c .
$$

Since, $x$ is curve of $\mathbb{E}^{n}$, then it satisfies the equality (2). Thus, we get $m_{0}=c^{2} s$. Hence, substituting this value into (6) one can get,

$$
\begin{aligned}
1-c^{2} & =k_{1} m_{1}+\ldots+k_{i} m_{i}+\ldots+k_{n-1} m_{n-1}, \\
m_{1} & =c^{2} \int s k_{1}(s) d s \\
m_{i} & =c^{2} \int s k_{i}(s) d s,(2 \leq i \leq n-1) .
\end{aligned}
$$

Consequently, we obtain the desired result.

\section{Competing interests}

The authors declare that they have no competing interests.

\section{Authors' contributions}

All authors have contributed to all parts of the article. All authors read and approved the final manuscript.

\section{References}

[1] L.R. Bishop, There is more than one way to frame a curve, Amer. Math. Monthly 82(3)(1975) 246-251.

[2] S. Büyükkütük, G. Öztürk, Constant ratio curves according to Bishop frame in Euclidean 3-space $\mathbb{E}^{3}$, Gen. Math. Notes $28(1)$ (2015) 81-91.

[3] S. Büyükkütük, G. Öztürk, Constant ratio curves according to parallel transport frame in Euclidean 4-space $\mathbb{E}^{4}$, New Trends in Mathematical Sciences 4(3) (2015) 171-178.

[4] B.Y. Chen, Constant ratio hypersurfaces, Soochow J. Math. 28 (2001) 353-362.

[5] B.Y. Chen, When does the position vector of a space curve always lies in its rectifying plane?, Amer. Math. Monthly 110 (2003) $147-152$. 
[6] B.Y. Chen, Geometry of Warped Products as Riemannian Submanifolds and Related Problemsc, Soochow Journal of Mathematics, 28(2) (2002) 125-156.

[7] B.Y. Chen, More on convolution of Riemannian manifolds, Beitrage Algebra und Geom. 44 (2003) 9-24.

[8] S. Cambie, W. Geomans, I.V.D Bussche, Rectifying curves in the n-dimensional Euclidean space, Turk J. Math 40 (2016) $210-223$.

[9] H. Gluck, Higher curvatures of curves in Euclidean space, The American Mathematical Monthly 73(7) (1966) 699-704.

[10] S. Gürpınar, K. Arslan, G. Öztürk, A characterization of constant-ratio curves in Euclidean 3-space $\mathbb{E}^{3}$, Acta Universitatis Apulensis 44 (2015) 39-51.

[11] K. İlarslan and E. Nesovic, Some characterizations of rectifying curves in the Euclidean space $\mathbb{E}^{4}$, Turk. J. Math. 32 (2008) 21-30.

[12] İ. Kişi, G. Öztürk, Constant ratio curves according to Bishop frame in Minkowski 3-space $\mathbb{E}_{1}^{3}$, Facta Universitatis, Series: Mathematics and Informatics 30(4) (2015) 527-538.

[13] C.L. Terng, Lecture notes on curves and surfaces in $\mathbb{R}^{3}$, Preliminary Version and in Progress, April 2, 2003. 